\title{
MicroRNA-16 regulates lipopolysaccharide-induced inflammatory factor expression by targeting TLR4 in normal human bronchial epithelial cells
}

\author{
XIAOBO LI, QIAN CHU and HUAQI WANG \\ Department of Respiratory and Critical Care Medicine, The First Affiliated Hospital \\ of Zhengzhou University, Zhengzhou, Henan 450052, P.R. China
}

Received November 6, 2020; Accepted May 4, 2021

DOI: $10.3892 /$ etm.2021.10414

\begin{abstract}
Acute lung injury (ALI) is mainly caused by inflammation and is associated with high mortality rates. Emerging evidence has suggested that microRNAs (miRNAs or miRs) serve a significant function in ALI. However, the fundamental mechanism underlying ALI remain to be fully elucidated. Although miR-16 has been reported to be involved in the occurrence and development of a number of diseases its association with ALI has not been previously investigated. Therefore, the present study aimed to explore the role of miR-16 in the lipopolysaccharide (LPS)-induced ALI model. The expression levels of tumor necrosis factor $\alpha$ (TNF- $\alpha$ ), interleukin (IL)-1 $\beta$ and IL- 6 were measured by ELISA in the blood samples of rats with ALI and in the normal human bronchial epithelial (NHBE) cell line. The role of miR-16 in inflammation was evaluated using gene overexpression and silencing experiments in NHBE cells by reverse transcription-quantitative PCR. In addition, the expression levels of inflammatory factors TNF- $\alpha$, IL-1 $\beta$ and IL- 6 were also determined using ELISA. The potential interaction between miR-16 and TLR4 was assessed using bioinformatics analysis by the TargetScan database and then verified in $293 \mathrm{~T}$ cells using luciferase reporter assay. The expression of miR-16 was notably decreased in the lung tissues of rats with LPS-induced ALI compared with the PBS treated-group. Additionally, the levels of the proinflammatory cytokines TNF- $\alpha$, IL- $1 \beta$ and IL- 6 were reduced following transfection of NHBE cells with miR-16 mimics compared with those in the miR-negative control group. Western blot analysis revealed that miR-16 overexpression could downregulate TLR4 expression in NHBE cells compared with that in the miR-NC group. Luciferase reporter assay confirmed
\end{abstract}

Correspondence to: Professor Huaqi Wang, Department of Respiratory and Critical Care Medicine, The First Affiliated Hospital of Zhengzhou University, 1 Jianshe East Road, Zhengzhou, Henan 450052, P.R. China

E-mail: wanghuaqi2004@126.com

Key words: toll-like receptor 4, acute lung injury, microRNA-16, lipopolysaccharide that TLR4 may be directly targeted by miR-16. The effect of miR-16 on TLR4 was rescued in NHBE cells following treatment with LPS. Overall, these aforementioned findings suggest that miR-16 may serve a protective role against LPS-mediated inflammatory responses in NHBE cells by regulating TLR4, where this mechanism may be considered to be a novel approach for treating ALI in the future.

\section{Introduction}

Acute lung injury (ALI) is a common life-threatening inflammatory condition that is associated with high morbidity and mortality rates in addition to high costs (1), with $>3$ million ARDS cases and 75,000 deaths annually worldwide $(2,3)$. The pathogenesis of ALI is highly complex. A number of studies have suggested that pulmonary edema and the hyperactivation of the innate immune response are closely associated with the onset of ALI $(4,5)$. Environmental and internal factors such as infection and sepsis (6), can mediate tissue inflammation and subsequent damage by inducing the secretion of inflammatory factors, including interleukin (IL)-1 $\beta$, IL-6, IL-8 and tumor necrosis factor- $\alpha$ (TNF- $\alpha$ ). Dysregulation of this process can eventually lead to alveolar-capillary barrier disruption and pulmonary edema $(7,8)$. MicroRNAs (miRNAs or miRs) are a type of small, non-coding, single-stranded RNAs that are 17-24 nucleotides in length and can modulate the expression of target genes on a post-transcriptional level $(9,10)$. It has been reported that miRs serve an important role in the occurrence and progression of ALI (11). Toll-like receptor 4 (TLR4) is a member of the conserved type I transmembrane proteins, which was first identified in microbes in 1980 (12). TLR4 mainly mediates the recognition of pathogens to initiate the inflammation signaling pathway (13). Several members of these proteins have since been found in mammals, including and humans $(14,15)$. Among them, TLR4 is a receptor that can recognize lipopolysaccharides (LPS) and plays a pivotal role in mediating lung injury (16). Upon activation, this upregulated state can then be modulated by several miRs, including miR-140, miR-181a and miR-182, via a negative feedback mechanism to inhibit inflammation (17-19)

Ventilation therapy approaches, such as mechanical ventilation with the use of low tidal volumes, have been developed for treating ALI (19). This method is designed to protect the 
lungs from excessive mechanical burden (19). It has also been documented that intratracheal installation of primary rat alveolar type II (ATII) cells in a rat ALI model can attenuate lung injury (20). However, the mechanistic details underlying the pathogenesis of ALI has not been fully elucidated. It was previously found that upregulation miR-16 can inhibit the inflammatory response in a ALI model of mouse (21). Therefore, the present study was undertaken to investigate whether miR-16 upregulation can attenuate the ALI-induced inflammatory responses. The role of miR-16 was investigated in a LPS-induced rat model of ALI, which aimed to uncover its underlying mechanisms of action and determine whether it is a potential target for the clinical therapy of ALI.

\section{Materials and methods}

Bioinformatics analysis. TargetScan database (release no. 7.2; https://www.targetscan.org/vert_72/) was used to calculate the prediction of targets of miR-16.

Animals. In total, 10 8-week-old female Sprague-Dawley rats weighing $240 \pm 20 \mathrm{~g}$ were purchased from Beijing Vital River Laboratory Animal Technology Co., Ltd and were kept in $23 \pm 2^{\circ} \mathrm{C}$ with a $12-\mathrm{h}$ light/dark cycle, $55-60 \%$ humidity, with ad libitum access to food and water. All the animal protocols (approval no. 2019-KY-005) were approved by The Ethics Committee of Zhengzhou University, with all animal experiments performed according to the National Institutes of Health Guidelines for the Care and Use of Laboratory Animals (GB/T 35892-2018).

Establishment of LPS-induced lung injury rat model. LPS (Sigma-Aldrich; Merck KGaA) was diluted in PBS to a final concentration of $10 \mathrm{mg} / \mathrm{ml}$. Rats were randomly divided into two groups. Rats in the LPS group $(n=5)$ were administered LPS $(0.1 \mathrm{mg} / \mathrm{kg})$ through tracheal intubation after anesthesia using isoflurane inhalation at a dose of 4 and $2 \%$ for induction and maintenance, respectively, at a gas flow rate of $0.25 \mathrm{l} / \mathrm{min}$ (22). Rats in the control group were treated with the same volume of PBS alone. At $6 \mathrm{~h}$ following the establishment of the LPS-induced lung injury model, all rats were euthanized with intraperitoneal injection of $200 \mathrm{mg} / \mathrm{kg}$ sodium pentobarbital followed by cervical dislocation. Subsequently, peripheral blood and the lung tissues were collected from all rats.

Cell culture and transfection. The NHBE(cat.no.PCS-300-010) normal human bronchial epithelial cell line and 293T cells were purchased from the American Type Culture Collection. Cells were cultured in DMEM (Thermo Fisher Scientific, Inc.) supplemented with $10 \%$ FBS (Thermo Fisher Scientific, Inc.) and $1 \%$ penicillin at $37^{\circ} \mathrm{C}$ in a humified atmosphere of $5 \% \mathrm{CO}_{2}$. miR-16 expression was tested in NHBE cells which was treated with LPS $(0,1,2,3,4,8$ and $16 \mu \mathrm{g} / \mathrm{ml})$ for $12 \mathrm{~h}$ at $37^{\circ} \mathrm{C}$ by reverse transcription-quantitative PCR (RT-qPCR). In addition, miR-16 expression was tested in NHBE cells that were incubated with $2 \mu \mathrm{g} / \mathrm{ml}$ LPS for a range of indicated time durations $(0,6,12,24,36$ and $48 \mathrm{~h})$ at $37^{\circ} \mathrm{C}$ by RT-qPCR.

The group method of NHBE cells as follows: i) miR-NC group; ii) miR-NC + LPS group, where the cells were transfected with miR-NC and then treated with $2 \mu \mathrm{g} / \mathrm{ml} \mathrm{LPS}$; iii) miR-16 mimic group, where cells were transfected with the miR-16 mimic; iv) miR-16 mimic + LPS group, where cells were transfected with the miR-16 mimic and then treated with $2 \mu \mathrm{g} / \mathrm{ml}$ LPS; v) si-NC group; vi) si-NC + LPS group, where the $\mathrm{NHBE}$ cells were transfected with si-NC and then treated with $2 \mu \mathrm{g} / \mathrm{ml}$ LPS; vii) si-TLR4 group; viii) si-TLR4 + LPS group, where NHBE cells were transfected with si-TLR4 and then treated with $2 \mu \mathrm{g} / \mathrm{ml} \mathrm{LPS}$; and ix) miR-16 mimic + pcDNA TLR4 + LPS group, where cells were transfected with miR-16 mimic and pcDNA-TLR4 and then treated with $2 \mu \mathrm{g} / \mathrm{ml} \mathrm{LPS.}$ All transfection experiments lasted $48 \mathrm{~h}$.

Specific small interfering RNAs (siRNAs) targeting TLR4, miR-16 mimics and negative control (NC) mimics $(50 \mathrm{nM}$; Shanghai GenePharma Co., Ltd.) were transfected into NHBE cells for 48 h using Lipofectamine ${ }^{\circledR} 2000$ (Invitrogen; Thermo Fisher Scientific, Inc.) according to the manufacturer's protocols. The sequences were as follows: MiR-16 mimics sense, 5'-UAGCAGCACGUAAAUAUUGGCG-3' and antisense, 5'-CGCCAAUAUUUACGUGCUGCUA-3'; miR-NC sense, UUCUCCGAACGUGUCACGUTT-3' and antisense, 5'-ACGUGACACGUUCGGAGAATT-3'; TLR4 siRNA sense, 5'-GGACAGCUUAUAACCUUAATT-3' and antisense, 5'-UUAAGGUUAUAAGCUGUCCTT-3' and siRNA-NC sense, 5'-UUCUCCGAACGUGUCACGUTT-3' and antisense, 5'-ACGUGACACGUUCGGAGAATT-3'. Total RNA was extracted from NHBE cells using the TRIzol ${ }^{\circledR}$ reagent (Invitrogen; Thermo Fisher Scientific, Inc.) before the cDNA was obtained by reverse transcription using the random primer (cat. no. B300818-0100; Sangon Biotech Co., Ltd.) and 5X AMV RT Buffer (cat. no. B610020-0500; Sangon Biotech Co., Ltd.), using the temperature protocol of $42^{\circ} \mathrm{C}$ for $60 \mathrm{~min}$ and $72^{\circ} \mathrm{C}$ for $10 \mathrm{~min}$. The TLR4 target was obtained by PCR (Direct PCR Kit; cat. no. B639289; Sangon Biotech Co., Ltd.) using TLR4-specific primers (TLR4 forward, 5'-AAAAGC TTGCCACCATGATGTCTGCCTCGCGCCTGG-3' and reverse, 5'-AAGGATCCTCAGATAGATGTTGCTTCCTGC-3'). The temperature protocol was $95^{\circ} \mathrm{C}$ for $30 \mathrm{sec}$, followed by 40 cycles of $95^{\circ} \mathrm{C}$ for $5 \mathrm{sec}$ and $60^{\circ} \mathrm{C}$ for $34 \mathrm{sec}$. The whole sequence of TLR4 was then sub-cloned into the pcDNA.3.1 vector (Shanghai GenePharma Co., Ltd.) via the HindIII and BamHI restriction sites to construct the TLR4 overexpression plasmid, which was also transfected into NHBE cells using Lipofectamine 2000 (17).

Histological analysis. For histological analysis, the lung tissues from PBS- or LPS-treated rats were fixed with $4 \%$ paraformaldehyde overnight at room temperature and embedded with paraffin. Subsequently, $4-\mu \mathrm{m}$ sections of the lung tissues were dehydrated in an ascending ethanol gradient and then xylene and were stained with hematoxylin and eosin (H\&E) (23). At room temperature, the samples were stained with hematoxylin for $5 \mathrm{~min}$ and eosin for $1 \mathrm{~min}$. Each slide was observed under light microscopy at x200 magnification. Representative images for each group are shown.

ELISA. The secretion levels of TNF- $\alpha$ (cat. no. E-EL-R0019c), IL-1 $\beta$ (cat. no. E-EL-H0149c) and IL-6 (cat. no. E-EL-H0102c) were determined using the corresponding ELISA kits (Elabscience Biotechnology Co., Ltd.) in peripheral blood samples of rats and cell culture medium of NHBE cells. 
All procedures were performed independently in triplicate according to manufacturer's protocol.

Reverse transcription-quantitative PCR (RT-qPCR). Total RNA was extracted from the lung tissues of rats or NHBE cells using the TRIzol ${ }^{\circledR}$ reagent (Invitrogen; Thermo Fisher Scientific, Inc.). For miRNA analysis, cDNA was synthesized by M-MLV reverse transcriptase with a special reverse transcription primer for miRNAs (PrimeScript ${ }^{\mathrm{TM}}$ 1st Strand cDNA Synthesis Kit; cat. no. 6110A; Takara Bio, Inc.) using the protocol of $30^{\circ} \mathrm{C} 10 \mathrm{~min}, 42^{\circ} \mathrm{C} 60 \mathrm{~min}$ and $95^{\circ} \mathrm{C}$ for $5 \mathrm{~min}$. For mRNA analysis, $c D N A$ was synthesized using a PrimeScript ${ }^{\mathrm{TM}}$ RT reagent kit (cat. no. RR037A; Takara Bio, Inc.) using the protocol of $16^{\circ} \mathrm{C}$ for $30 \mathrm{~min}, 42^{\circ} \mathrm{C}$ for $30 \mathrm{~min}$ and $85^{\circ} \mathrm{C} 10 \mathrm{~min}$. For miRNAs, qPCR was performed using Hairpin-it ${ }^{\mathrm{TM}}$ miRNAs qPCR Quantitation Kit (cat. no. QPM-010, Shanghai GenePharma Co., Ltd.). The primer sequences of miR-16 used were the following: Forward, 5'-CGCGCTAGCAGC ACGTAAAT-3' and reverse, 5'-GTGCAGGGTCCGAGGT-3'; U6 forward, 5'-CTCGCTTCGGCAGCACA-3' and reverse, 5'-AACGCTTCACGAATTTGCGT-3'. For mRNA, qPCR was performed using the LightCycler ${ }^{\circledR}$ RNA Master SYBR Green I (cat. no. 3064760001; Roche Diagnostics). The sequences of the primers were as follows: TLR4 forward, 5'-GGCTCC TGATGCAAGATGCCCCT-3' and reverse, 5'-CTGCCTTGA ATACCTTCACACGT-3'; TNF- $\alpha$ forward, 5'-CTGGGACAG TGACCTGGACT-3' and reverse, 5'-GCACCTCAGGGAAGA GTCTG-3'; IL-1 $\beta$ forward, 5'-CCTCCTTGCCTCTGATGG-3' and reverse, 5'-AGTGCTGCCTAATGTCCC-3'; IL-6 forward, 5'-AGTTGCCTTCTTGGGACTGA-3' and reverse, 5'-TCC ACGATTTCCCAGAGAAC-3' and GAPDH forward, 5'-CTG AGCACCAGGTGGTCTC-3' and reverse, 5'-CATGACAAG GTGCGGCTCC-3'. The thermocycling conditions were $95^{\circ} \mathrm{C}$ for $30 \mathrm{sec}$, followed by 40 cycles of $95^{\circ} \mathrm{C}$ for $5 \mathrm{sec}$ and $60^{\circ} \mathrm{C}$ and 34 sec. qPCR was performed using an ABI 7500 Fast Real-time PCR system (Applied Biosystems; Thermo Fisher Scientific, Inc.). Relative expression of miR-16 was normalized to that of U6, whereas the relative mRNA expression was normalized to GAPDH, both using the $2^{-\Delta \Delta \mathrm{Cq}}$ method (24-26).

Luciferase reporter assay. 293T cells ( $1 \times 10^{5}$ cells) were co-transfected with $10 \mathrm{pmol} / \mathrm{ml} \mathrm{miR}-16$ mimics or miR-NC and pmirGLO3-TLR4-3'UTR-wild-type (Wt) or pmirGLO3-TLR4-3'UTR-mutant (MUT) $(0.2 \mathrm{pmol} / \mathrm{ml}$ all from Shanghai GenePharma Co., Ltd.) by Lipofectamine 2000. Following incubation for $48 \mathrm{~h}$ at $37^{\circ} \mathrm{C}$, the luciferase activity was measured using the Dual-Luciferase ${ }^{\circledR}$ Reporter Assay System (Promega Corporation) according to the manufacturer's protocols. The results of firefly luciferase activity were normalized to the Renilla luciferase activity.

Western blot analysis. Total proteins of NHBE cells were extracted using a protein extraction kit (NE-PER ${ }^{\mathrm{TM}}$ Nuclear and Cytoplasmic Extraction Reagents; Pierce, Thermo Fisher Scientific, Inc.) and the protein concentration was measured using bicinchoninic acid protein assay reagent (Beyotime Institute of Biotechnology). A 10\% SDS-PAGE gel was prepared with $20 \mu \mathrm{g}$ protein samples loaded in each lane. The samples were mixed with the loading buffer and boiled at $100^{\circ} \mathrm{C}$ for $5 \mathrm{~min}$. The protein on the gel was then transferred to a nitrocellulose membranes and blocked with $5 \%$ skimmed milk powder at room temperature $1 \mathrm{~h}$. GAPDH primary antibody (cat. no. ab70699; 1:5,000; Abcam) was chosen as the internal reference. The membranes were first probed with the anti-TLR4 mouse polyclonal antibody incubated at $4^{\circ} \mathrm{C}$ overnight (cat. no. sc-293072; dilution 1:1,500; Santa Cruz Biotechnology, Inc.) and then with a corresponding horseradish peroxidase-conjugated secondary antibody (cat. no. sc-525409; 1:5,000; Santa Cruz Biotechnology, Inc.) at room temperature for $1.5 \mathrm{~h}$. An enhanced chemiluminescence kit (EMD Millipore) was used for visualization of the results and quantification of the bands was performed using the Image J software (v1.8.0; National Institutes of Health).

Statistical analysis. All statistical analyses were performed using unpaired Student's t-test for comparisons between two groups. One-way ANOVA and Tukey's post hoc test were used for multiple group comparisons using the SPSS software (version 17.0; SPSS, Inc.) Data are expressed as the mean \pm SD from three independent experiments performed in triplicate. $\mathrm{P}<0.05$ was considered to indicate a statistically significant difference.

\section{Results}

miR-16 expression is downregulated in the lung tissues of ALI rats and LPS-induced NHBE cells. An ALI rat model was established by LPS, whilst animals treated with PBS served as control. The lung tissues from the LPS group showed lung injury, including hemorrhage, interstitial edema and infiltration of inflammatory cells (Fig. 1A). Subsequent RT-qPCR analysis showed that the expression of miR-16 was significantly decreased in the lung tissues of rats treated with LPS compared with that in control tissues treated with PBS (Fig. 1B). ELISA results revealed that the secretion levels of IL-1 $\beta$, TNF- $\alpha$ and IL- 6 were significantly increased in the peripheral blood of LPS-treated rats compared with those in the control PBS group (Fig. 1C-E).

In NHBE cells treated with different concentrations (Fig. 1F) of LPS for different time periods (Fig. 1G), the expression levels of miR-16 were markedly decreased compared with those in the 0 group. These data suggested a potential association between the expression of miR-16 and the LPS-induced inflammatory response in the lung.

miR-16 attenuates the secretion of proinflammatory cytokines by LPS-induced NHBE cells. To investigate the role of miR-16 in ALI, NHBE cells were transfected with either miR-16 mimics or miR-NC, which significantly increased miR-16 expression compared with that in the miR-NC group (Fig. 2A). The secretion levels of IL-1 $\beta$, TNF- $\alpha$ and IL-6 were determined by ELISA in cell culture supernatant treated for $12 \mathrm{~h}$ with LPS $(2 \mu \mathrm{g} / \mathrm{ml})$ and control cells after transfection. The results demonstrated that the secretion levels of proinflammatory cytokines were markedly reduced in cells in the miR-16 mimic + LPS group compared with those in the miR-NC + LPS group (Fig. 2B-D). Additionally, mRNA expression levels of TNF- $\alpha$, IL-1 $\beta$ and IL- 6 were also assessed by RT-qPCR in NHBE cells (Fig. 2E-G). Consistent with the ELISA data, the expression levels of the proinflammatory 
A

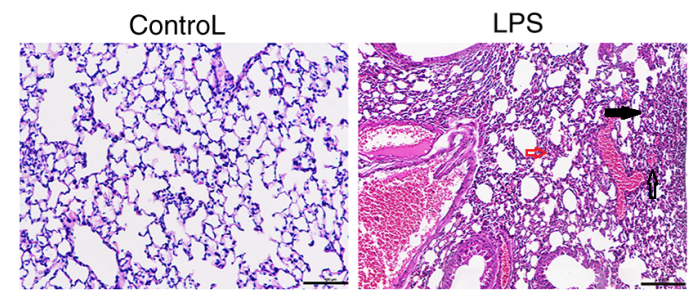

$\mathrm{B}$

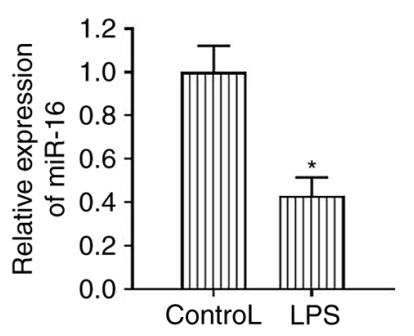

C

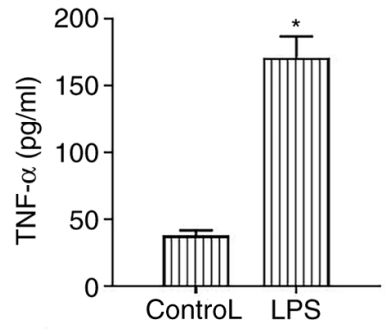

D

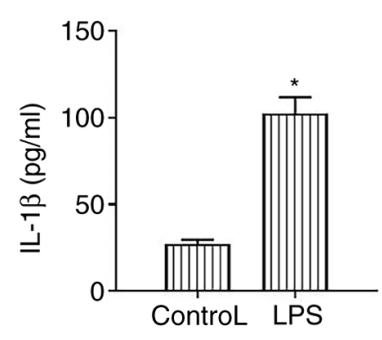

E

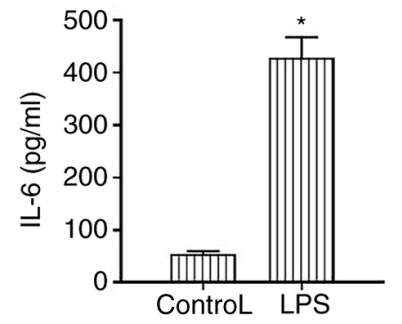

$\mathrm{F}$

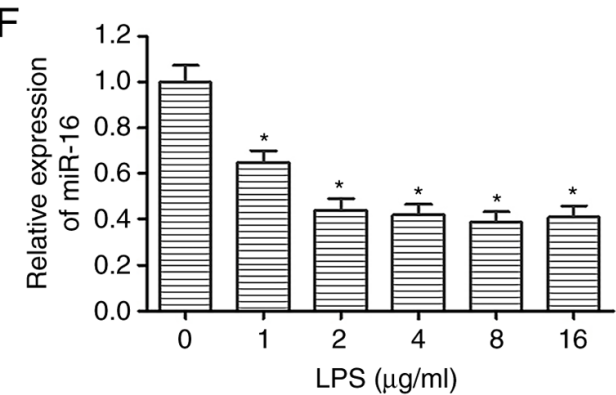

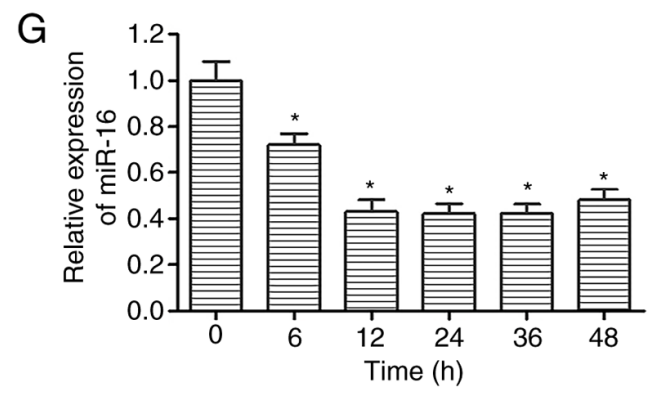

Figure 1. Treatment of rats and NHBE cells with LPS downregulates miR-16 expression. (A) Hematoxylin and eosin staining revealed histological differences between the lung tissues from rats in the LPS and control groups. The lung tissues from the LPS group showed lung injury, including hemorrhage (clear arrow), interstitial edema (red arrow) and infiltration of inflammatory cells (black arrow). Scale bar, $100 \mu \mathrm{m}$. (B) Differences in miR-16 expression between rats in the LPS and control groups were assessed by reverse transcription-quantitative PCR. The levels of (C) TNF- $\alpha$, (D) IL- $1 \beta$ and (E) IL- 6 were measured in rats treated with LPS or PBS (control) using ELISA. "P<0.05 vs. Control. (F) miR-16 expression was decreased following treatment of NHBE cells with increasing concentrations of LPS $(0,1,2,3,4,8$ and $16 \mu \mathrm{g} / \mathrm{ml})$ for $12 \mathrm{~h}$ as assessed by reverse transcription-quantitative PCR. ${ }^{\mathrm{P}}<0.05$ vs. 0 group. (G) miR-16 expression was gradually reduced in NHBE cells incubated with $2 \mu \mathrm{g} / \mathrm{ml}$ LPS for a range of indicated time durations $(0,6,12,24,36$ and $48 \mathrm{~h})$ as assessed by reverse transcription-quantitative PCR. "P<0.05 vs. 0 group. "P<0.05. LPS, lipopolysaccharide; ALI, acute lung injury; TNF- $\alpha$, tumor necrosis factor $\alpha$; IL, interleukin; NHBE, normal human bronchial epithelial; NC, negative control; miR, microRNA.

cytokines were also decreased in the miR-16 mimic + LPS group compared with those in the miR-NC + LPS group. These findings suggest that miR-16 can negatively regulate the expression of inflammatory factors. However, whether miR-16 can directly target these inflammatory cytokines requires further investigation.

TLR4 is directly targeted by miR-16. A conserved binding region between the 3'-UTR of TLR4 mRNA and miR-16 was identified by TargetScan analysis (Fig. 3A). It was therefore hypothesized that miR-16 could directly target TLR4. Western blot analysis revealed that TLR4 was downregulated in NHBE cells transfected with miR-16 mimics compared with that in cells transfected with miR-NC (Fig. 3C). To verify if TLR4 can be directly targeted by miR-16 in vitro, a luciferase reporter assay was performed. The data revealed that the luciferase activity of the pmirGLO3-TLR4-3'-UTR-Wt reporter was significantly suppressed in the miR-16 mimics group compared with the that in miR-NC group (Fig. 3B). However, miR-16 mimic had no effect on the luciferase activity of the pmirGLO3-TLR4-3'-UTR-MUT construct (Fig. 3B). These findings indicated that miR-16 could directly target TLR4 3'-UTR to suppress its expression on the post-transcriptional level.

TLR4 silencing alleviates LPS-induced inflammatory responses. To further evaluate the effect of TLR4 on LPS-induced inflammatory responses, cells were transfected with specific siRNAs targeting TLR4. A shown in Fig. 4A, transfection of cells with si-TLR4 significantly downregulated TLR4 protein expression compared with that in cells transfected with si-NC. TLR4 knockdown also significantly attenuated the LPS-induced secretion of IL- $1 \beta$, TNF- $\alpha$ and IL- 6 by NHBE cells compared with those in LPS-treated cells transfected with si-NC (Fig. 4B-D). Therefore, these data suggested that TLR4 knockdown could reduce the production of proinflammatory cytokines.

miR-16 regulates inflammatory responses to LPS in NHBE cells by targeting TLR4. Subsequently, TLR4 was overexpressed in NHBE cells that were also transfected with miR-16 mimics to investigate whether miR-16 could regulate the inflammatory 

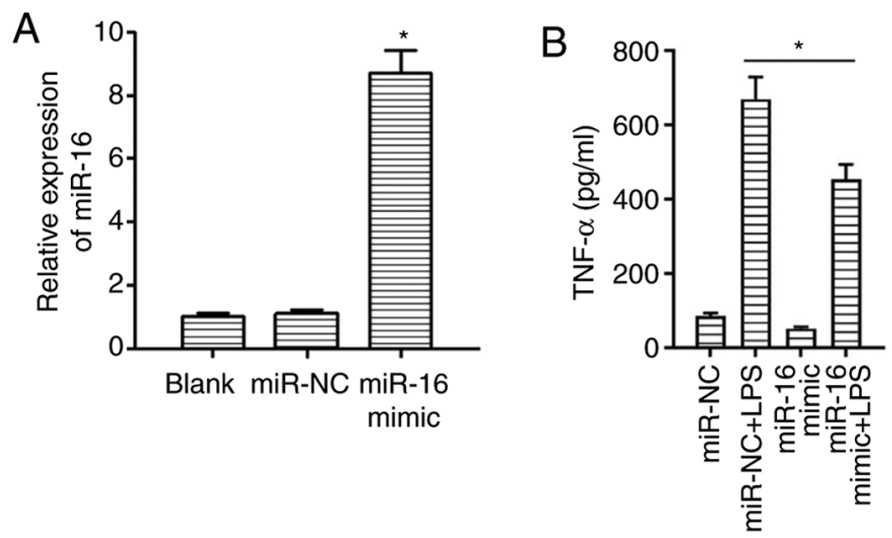

F

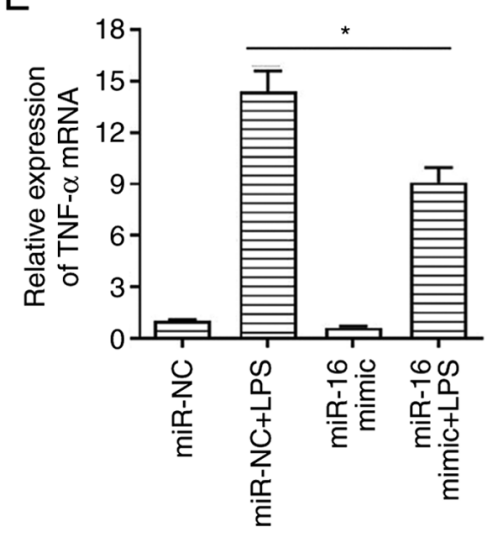

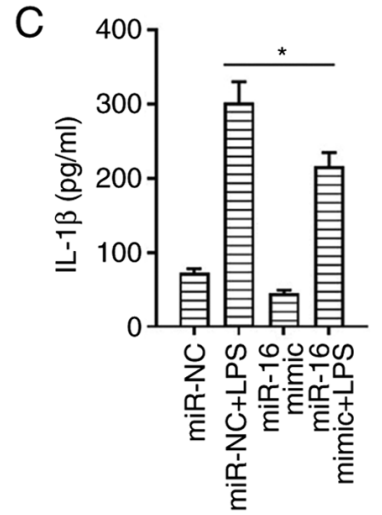
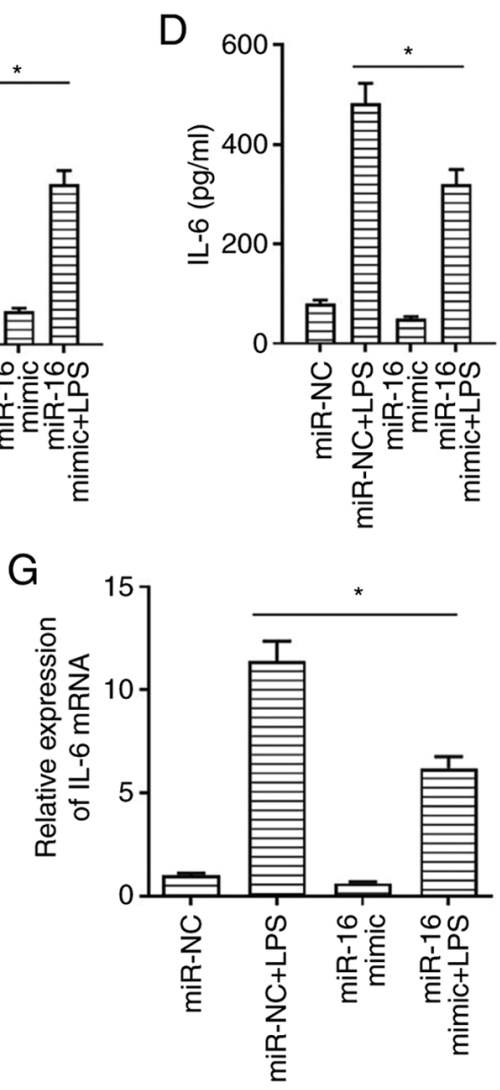

Figure 2. miR-16 overexpression attenuates LPS-induced secretion of IL-1 $\beta$, TNF- $\alpha$ and IL-6. NHBE cells were treated with $2 \mu \mathrm{g} / \mathrm{ml} \mathrm{LPS}$ for $12 \mathrm{~h}$ after transfection 48 h. (A) Transfection of NHBE cells with miR-16 mimics sufficiently overexpressed miR-16. "P<0.05 vs. miR-NC. Secretion of (B) TNF- $\alpha$, (C) IL-1 $\beta$ and (D) IL- 6 by NHBE cells was determined following cell transfection with miR-16 mimics for $48 \mathrm{~h}$ using ELISA. mRNA expression levels of (E) TNF- $\alpha$, (F) IL-1 $\beta$ and (G) IL-6 were measured in NHBE cells by reverse transcription-quantitative PCR. "P<0.05. LPS, lipopolysaccharide; TNF- $\alpha$, tumor necrosis factor $\alpha$; IL-1 $\beta$, interleukin $1 \beta$; NHBE, normal human bronchial epithelial; NC, negative control; miR, microRNA.

A

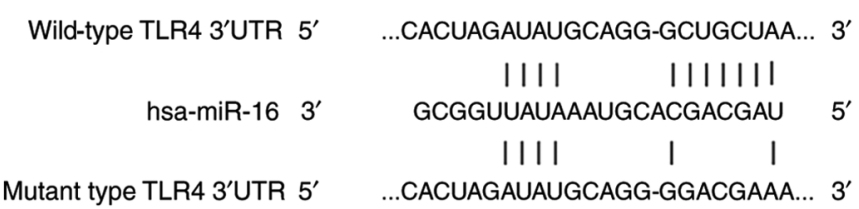

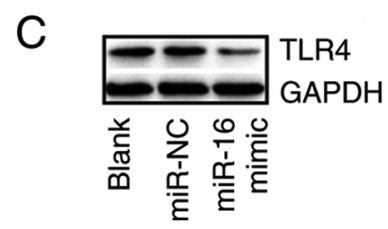

B

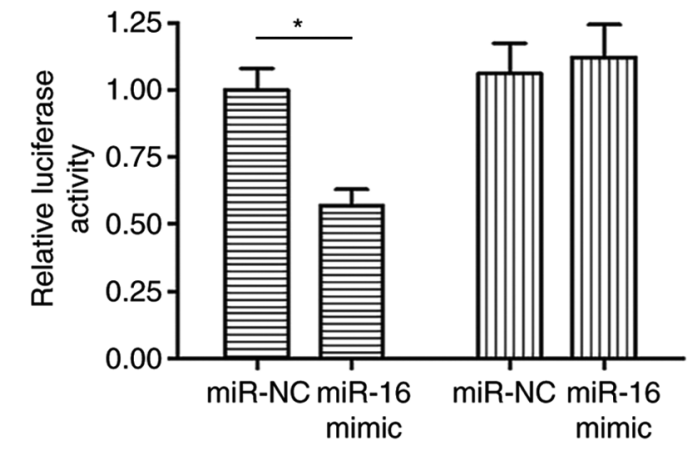

Figure 3. miR-16 directly targets TLR4. (A) A specific binding site of miR-16 was identified within the 3'-UTR of TLR4 using Targetscan bioinformatics analysis. (B) Luciferase reporter assay was used to verify if miR-16 could directly target the TLR4 3'-UTR in 293T cells. (C) Western blot analysis for TLR4 protein expression after miR-16 mimic transfection in NHBE cells. " $\mathrm{P}<0.05$ (compare with wild NC group). 3'-UTR, 3'-untranslated region; TLR4, toll-like receptor 4; miR, microRNA; NC, negative control.

responses by targeting TLR4. Following transfection with miR-16 mimics and or pcDNA3.1-TLR4, cells were treated with LPS $(2 \mu \mathrm{g} / \mathrm{ml})$ before the levels of the proinflammatory cytokines were measured by ELISA. As shown in Fig. 5A-C, cell treatment with LPS increased the secretion levels of IL-1 $\beta$, TNF- $\alpha$ and IL- 6 compared with those in the control, which were reversed by miR-16 overexpression compared with those in the LPS + miR-NC. However, the protective mechanism of miR-16 against this particular inflammatory response as reported by IL- $1 \beta$, TNF- $\alpha$ and IL- 6 levels were significantly negated following TLR4 overexpression. Strict transfection efficiency controls were also set up (Fig. S1). To conclude, 
A

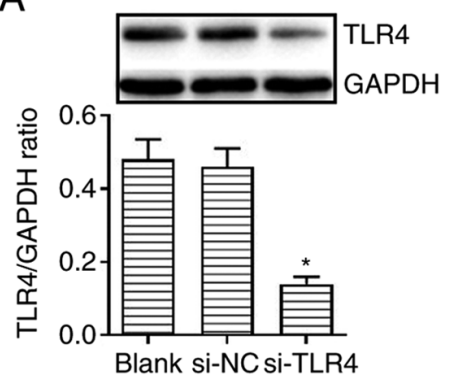

B

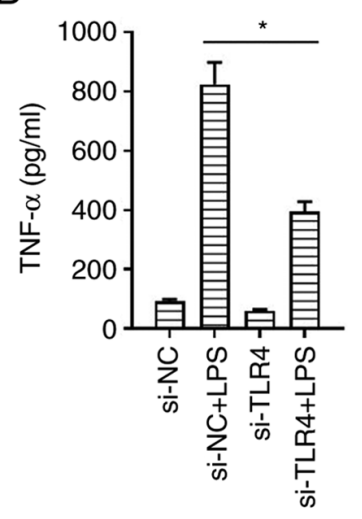

C

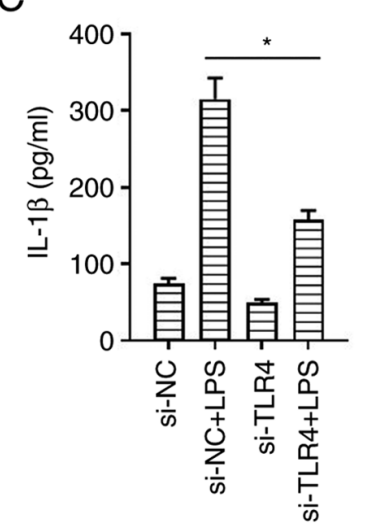

$\mathrm{D}$

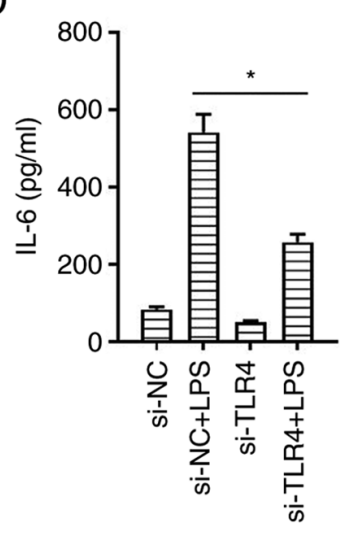

Figure 4. TLR4 knockdown alleviates LPS-induced inflammatory factors secretion from NHBE cells. (A) Western blot analysis for TLR4 protein expression after transfection with si-TLR4. The secretion levels of (B) TNF- $\alpha$, (C) IL-1 $\beta$ and (D) IL-6 were measured by ELISA following transfection of NHBE cells with si-TLR4 or si-NC for $48 \mathrm{~h}$. * P<0.05. LPS, lipopolysaccharide; TNF- $\alpha$, tumor necrosis factor $\alpha$; IL, interleukin; si, small interfering; NC, negative control; TLR4, toll-like receptor 4; NHBE, normal human bronchial epithelial.

A

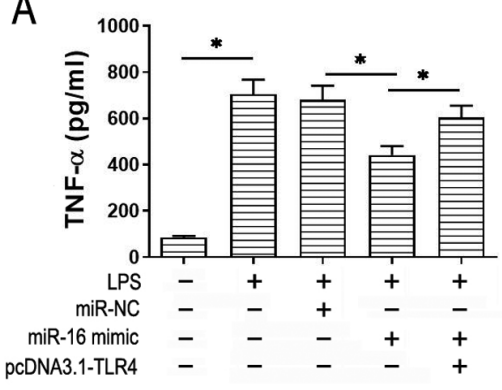

B

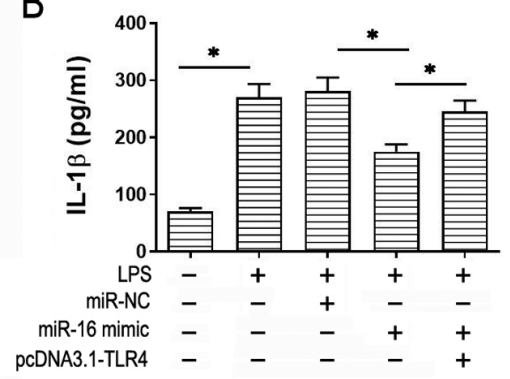

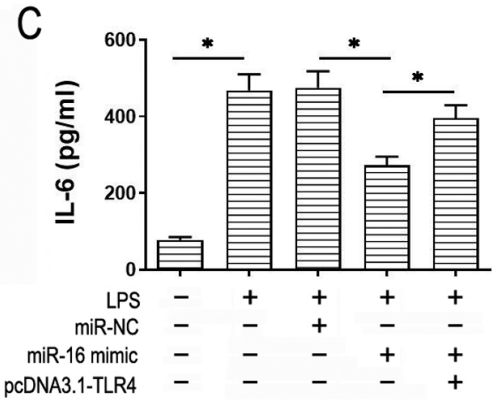

Figure 5. miR-16 exerts protective effects against ALI-induced inflammatory responses by modulating TLR4 expression. Secretion levels of (A) TNF- $\alpha$, (B) IL-1 $\beta$ and (C) IL-6 were assessed by ELISA after co-transfection and LPS treatment. * $<<0.05$. ALI, acute lung injury; TNF- $\alpha$, tumor necrosis factor $\alpha$; IL-1 $\beta$, interleukin $1 \beta$.

these aforementioned findings suggested that miR-16 may suppress the secretion of proinflammatory cytokines by targeting TLR4.

\section{Discussion}

ALI is an inflammatory pulmonary condition that is characterized by refractory hypoxemia and dyspnea, with substantial risk of mortality (3). However, the mechanism underlying ALI remains poorly understood (27). It has been previously reported that inflammatory disorders and alveolar barrier disruption are the main causes of ALI (28). Therefore, inhibiting inflammatory responses can be considered to effectively improve LPS-induced ALI (29). Acute respiratory distress syndrome (ARDS) is considered to be severe type of ALI $(3,30)$. In 2003 , a number of patients with severe acute respiratory syndrome (SARS) died from ARDS $(31,32)$. In addition, since the end of 2019, a severe pneumonia caused coronavirus disease 2019 (COVID-19) has become a global pandemic. This disease is caused by the SARS-coronavirus-2 (SARS-CoV-2), which is similar to SARS-CoV (33). The majority of patients with COVID-19 suffer from a series of systemic symptoms, which may progress into ARDS (34). However, a number of studies have shown that the TLR4 signaling pathway can control the progress of ALI $(32,35)$.
TLR4 serves an essential role in the immne and inflammatory response (36). TLR4 is not only involved in acquired immunity, but also in innate immunity (37). LPS is a component of the bacterial endotoxin that can activate TLR4 via the TLR4/myeloid differentiation primary response 88 or TLR4/TIR-domain-containing adapter-inducing interferon- $\beta$ /tumor necrosis factor receptor-associated factor 6 pathway to promote inflammation in the lungs (38). Therefore, IL-1 $\beta$, TNF- $\alpha$ and IL- 6 may be produced and aggravate ALI (27). Additionally, suppression of TLR4 may be associated with the alleviation of inflammatory reactions in a mouse lung injury model induced by LPS (39). Consistent with this hypothesis, it was previously demonstrated that dioscin (40), 25-hydroxycholesterol (41) and antagonists of the TLR4 signaling may alleviate LPS-induced ALI (42).

miRNAs have become a popular topic of scientific research, due to reports of their involvement in several biological processes, including cell apoptosis, proliferation and tumorigenesis (43). Therefore, it has been suggested that miRNAs may act as regulatory factors that can reduce the risk of a various diseases and improve the efficacy of therapy by regulating the expression of target mRNAs (44). For example, a previous study revealed that miR-302b could inhibit alveolar macrophages and epithelial cell inflammatory responses against bacterial infections by providing negative 
feedback to TLRs-mediated immunity (45). Another study reported that miR-203 inhibited ovarian tumor metastasis by targeting the transforming growth factor- $\beta$ pathway (46). Furthermore, miRNAs may be associated with the release of inflammatory cytokines. It has been documented that miR-182-5p (47), miR-106a (24) and miR-302b (45) upregulate the expression levels of inflammatory cytokines by targeting TLR4 to promote lung injury. miR-183-5p overexpression was previously reported to downregulate TLR4, which lead to the inhibition of inflammatory cytokine secretion (48). It has also been previously suggested that miR-16 may be used as a prognostic biomarker for chronic lymphocytic leukemia (CLL), since miR-16 downregulated bcl-2 expression to induce apoptosis in B-cell CLL (49). In addition, another study suggested that miR-16 may improve the prognosis of malignant mesothelioma, where after delivery of a miR-16 TargomiR, patients assessed by CT achieved disease control (50). Although some molecular mechanisms remain unknown, it is becoming well supported that miR-16 serves an significant role in the progression of several diseases. However, the role of miR-16 in LPS-induced ALI remains unclear.

A similar study has been recently published by Yang et al (21), which reported that upregulation of miR-16 can inhibit the expression of the nucleotide-binding oligomerization domain, leucine rich repeat and pyrin domain containing 3(NPRP3) inflammasome via TLR4 in A549 cells and a ALI model (21). However, this previous study did not perform rescue experiments to prove that TLR4 is an essential component, where miR-16 may regulate the NLRP3 inflammasome via other pathways. The present study used pcDNA3.1-TLR4 to confirm that TLR4 overexpression can reverse the downregulation of inflammatory cytokines caused by miR-16 overexpression. Also in the present study the ELISA results demonstrated that LPS-induced NHBE cells secreted increased levels of the inflammatory cytokines TNF- $\alpha$, IL-1 $\beta$ and IL- 6 . However, miR-16 overexpression reduced the levels of IL-1 $\beta$, TNF- $\alpha$ and IL- 6 , whilst this reduction in inflammatory cytokine secretion was negated when miR-16 and TLR4 were simultaneously overexpressed. RT-qPCR analysis revealed that miR-16 overexpression suppressed the expression of TLR4. Subsequently, the present study investigated the mechanism underlying the inhibitory effects of miR-16 on the inflammatory response. The results revealed that TLR4 downregulation downstream of miR-16 attenuated the inflammatory response in NHBE. Unfortunately, the protective effect of miR-16 was not verified in the animal experiments, which is a limitation of the present study. However, data from the present study appeared to be consistent with the data from the animal experiments previously performed by Yang et al (21), which provide support for the present findings.

To conclude, the present study demonstrated that miR-16 exerted its protective effects against ALI-induced inflammatory responses by modulating TLR4. The effective control of ALI remains challenging. miR-16 may provide reference for the clinical treatment of ALI, or even ARDS. Finally, although the present study demonstrated that miR-16 may play a key role in regulating LPS-induced ALI, further extensive scientific research is required before these findings will be applicable in clinical practice.

\section{Acknowledgements}

Not applicable.

\section{Funding}

The present study is supported by the project of National Natural Science Foundation of China (grant no. 81972182).

\section{Availability of data and materials}

The datasets used and/or analyzed during the current study are available from the corresponding author on reasonable request.

\section{Authors' contributions}

HW conceived and designed the experiments. XL and QC performed the experiments. XL and $\mathrm{HW}$ analyzed the data. XL and HW can authenticate the raw data in this study. All authors read and approved the final version of the manuscript.

\section{Ethics approval and consent to participate}

Animal protocols were approved by the Ethics Committee Of The First Affiliated Hospital Of Zhengzhou University (Zhengzhou, China) and all animal experiments were operated according to National Institutes of Health Guidelines for the Care and Use of Laboratory Animals.

\section{Patient consent for publication}

Not applicable.

\section{Competing interests}

The authors declare that they have no competing interests.

\section{References}

1. Wheeler AP and Bernard GR: Acute lung injury and the acute respiratory distress syndrome: A clinical review. Lancet 369: 1553-1564, 2007.

2. Fan E, Brodie D and Slutsky AS: Acute respiratory distress syndrome: Advances in diagnosis and treatment. JAMA 319: 698-710, 2018.

3. He YQ, Zhou CC, Yu LY, Wang L, Deng JL, Tao YL, Zhang F and Chen WS: Natural product derived phytochemicals in managing acute lung injury by multiple mechanisms. Pharmacol Res 163: 105224, 2021.

4. Matthay MA and Zimmerman GA: Acute lung injury and the acute respiratory distress syndrome: Four decades of inquiry into pathogenesis and rational management. Am J Respir Cell Mol Biol 33: 319-327, 2005.

5. Sahetya SK, Goligher EC and Brower RG: Fifty years of research in ARDS. setting positive end-expiratory pressure in acute respiratory distress syndrome. Am J Respir Crit Care Med 195: 1429-1438, 2017.

6. Deng JC and Standiford TJ: Growth factors and cytokines in acute lung injury. Compr Physiol 1: 81-104, 2011.

7. Grommes J, Morgelin M and Soehnlein O: Pioglitazone attenuates endotoxin-induced acute lung injury by reducing neutrophil recruitment. Eur Respir J 40: 416-423, 2012.

8. Johnston CJ, Finkelstein JN, Gelein R and Oberdorster G: Pulmonary cytokine and chemokine mRNA levels after inhalation of lipopolysaccharide in C57BL/6 mice. Toxicol Sci 46: 300-307, 1998. 
9. Martins HC and Schratt G: MicroRNA-dependent control of neuroplasticity in affective disorders. Transl Psychiatry 11: 263, 2021.

10. Zhao W, Sun Q, Yu Z, Mao S, Jin Y, Li J, Jiang Z, Zhang Y, Chen M, Chen P, et al: MiR-320a-3p/ELF3 axis regulates cell metastasis and invasion in non-small cell lung cancer via PI3K/Akt pathway. Gene 670: 31-37, 2018.

11. Ferruelo A, Penuelas $\mathrm{O}$ and Lorente JA: MicroRNAs as biomarkers of acute lung injury. Ann Transl Med 6: 34, 2018.

12. Nusslein-Volhard $C$ and Wieschaus E: Mutations affecting segment number and polarity in Drosophila. Nature 287: 795-801, 1980

13. Ciesielska A, Matyjek M and Kwiatkowska K: TLR4 and CD14 trafficking and its influence on LPS-induced pro-inflammatory signaling. Cell Mol Life Sci 78: 1233-1261, 2021.

14. Plociennikowska A, Hromada-Judycka A, Borzecka K and Kwiatkowska K: Co-operation of TLR4 and raft proteins in LPS-induced pro-inflammatory signaling. Cell Mol Life Sci 72 $557-581,2015$

15. Sukkar MB, Xie S, Khorasani NM, Kon OM, Stanbridge R, Issa R and Chung KF: Toll-like receptor 2, 3, and 4 expression and function in human airway smooth muscle. J Allergy Clin Immunol 118: 641-648, 2006.

16. Baumgarten G, Knuefermann P, Wrigge H, Putensen C, Stapel H, Fink K, Meyer R, Hoeft A and Grohé C: Role of Toll-like receptor 4 for the pathogenesis of acute lung injury in Gram-negative sepsis. Eur J Anaesthesiol 23: 1041-1048, 2006.

17. Jiang K, Guo S, Zhang T, Yang Y, Zhao G, Shaukat A, Wu H and Deng G: Downregulation of TLR4 by miR-181a provides negative feedback regulation to lipopolysaccharide-induced inflammation. Front Pharmacol 9: 142, 2018.

18. Yang J, Chen Y, Jiang K, Zhao G, Guo S, Liu J, Yang Y and Deng G: MicroRNA-182 supplies negative feedback regulation to ameliorate lipopolysaccharide-induced ALI in mice by targeting TLR4. J Cell Physiol 235: 5925-5937, 2020.

19. Acute Respiratory Distress Syndrome Network, Brower RG, Matthay MA, Morris A, Schoenfeld D, Thompson BT and Wheeler A: Ventilation with lower tidal volumes as compared with traditional tidal volumes for acute lung injury and the acute respiratory distress syndrome. N Engl J Med 342: 1301-1308, 2000.

20. Guillamat-Prats R, Puig F, Camprubi-Rimblas M, Herrero R, Serrano-Mollar A, Gómez MN, Tijero J, Matthay MA, Blanch L and Artigas A: Intratracheal instillation of alveolar type II cells enhances recovery from acute lung injury in rats. J Heart Lung Transplant 37: 782-791, 2018

21. Yang Y, Yang F, Yu X, Wang B, Yang Y, Zhou X, Cheng R, Xia S and Zhou X: MiR-16 inhibits NLRP3 inflammasome activation by directly targeting TLR4 in acute lung injury. Biomed Pharmacother 112: 108664, 2019.

22. McCarter SD, Mei SH, Lai PF, Zhang QW, Parker CH, Suen RS, Hood RD, Zhao YD, Deng Y, Han RN, et al: Cell-based angiopoietin-1 gene therapy for acute lung injury. Am J Respir Crit Care Med 175: 1014-1026, 2007.

23. Wu CT, Huang Y, Pei ZY, Xi X and Zhu GF: MicroRNA-326 aggravates acute lung injury in septic shock by mediating the NF-kappaB signaling pathway. Int J Biochem Cell Biol 101: 1-11, 2018.

24. Yang J, Chen Y, Jiang K, Yang Y, Zhao G, Guo S and Deng G: MicroRNA-106a provides negative feedback regulation in lipopolysaccharide-induced inflammation by targeting TLR4. Int J Biol Sci 15: 2308-2319, 2019.

25. Chungen Y, Dongfang Z and Guoyuan X: MicroRNA-146a protects against ischemia/reperfusion liver injury through inhibition of toll-like receptor 4 signaling pathway in rats. Transplant Proc 52: 1007-1013, 2020.

26. Livak KJ and Schmittgen TD: Analysis of relative gene expression data using real-time quantitative PCR and the 2(-Delta Delta C(T)) method. Methods 25: 402-408, 2001

27. Butt Y, Kurdowska A and Allen TC: Acute lung injury: A clinical and molecular review. Arch Pathol Lab Med 140: 345-350, 2016

28. Xu B, Chen SS, Liu MZ, Gan CX, Li JQ and Guo GH: Stem cell derived exosomes-based therapy for acute lung injury and acute respiratory distress syndrome: A novel therapeutic strategy. Life Sci 254: 117766, 2020.

29. Matthay MA, Ware LB and Zimmerman GA: The acute respiratory distress syndrome. J Clin Invest 122: 2731-2740, 2012.

30. Gao XQ, Li YF and Jiang ZL: Impact of statins on ALI/ARDS: A meta-analysis. Pulm Pharmacol Ther 39: 85-91, 2016.

31. Shah D, Das P, Acharya S, Agarwal B, Christensen DJ, Robertson SM and Bhandari V: Small immunomodulatory molecules as potential therapeutics in experimental murine models of acute lung injury (ALI)/acute respiratory distress syndrome (ARDS). Int J Mol Sci 22: 2573, 2021
32. Imai Y, Kuba K, Neely GG, Yaghubian-Malhami R, Perkmann T, van Loo G, Ermolaeva M, Veldhuizen R, Leung YH, Wang $\mathrm{H}$, et al: Identification of oxidative stress and Toll-like receptor 4 signaling as a key pathway of acute lung injury. Cell 133: 235-249, 2008

33. Sun J, Zhuang Z, Zheng J, Li K, Wong RL, Liu D, Huang J, He J, Zhu A, Zhao J, et al: Generation of a Broadly useful model for COVID-19 pathogenesis, vaccination, and treatment. Cell 182: 734-743.e5, 2020.

34. Pascarella G, Strumia A, Piliego C, Bruno F, Del Buono R, Costa F, Scarlata S and Agrò FE: COVID-19 diagnosis and management: A comprehensive review. J Intern Med 288: 192-206, 2020.

35. Zhou M, Zhang Y, Tang R, Liu H, Du M, Gao Z, Ji Z and Fang H: HMGB1/TLR4 signaling affects regulatory $T$ cells in acute lung injury. J Inflamm Res 14: 1551-1561, 2021.

36. Zhang W, Zhuang N, Liu X, He L, He Y, Mahinthichaichan P, Zhang H, Kang Y, Lu Y, Wu Q, et al: The metabolic regulator Lamtor5 suppresses inflammatory signaling via regulating mTOR-mediated TLR4 degradation. Cell Mol Immunol 17: 1063-1076, 2020.

37. Knoops B, Becker S, Poncin MA, Glibert J, Derclaye S, Clippe A and Alsteens D: Specific interactions measured by AFM on living cells between peroxiredoxin-5 and TLR4: Relevance for mechanisms of innate immunity. Cell Chem Biol 25: 550-559. e3, 2018.

38. Martin TR and Wurfel MM: A TRIFfic perspective on acute lung injury. Cell 133: 208-210, 2008.

39. Feng L, Yang N, Li C, Tian G, Wang J, Dong ZB, Jia XB and Di LQ: Pudilan xiaoyan oral liquid alleviates LPS-induced respiratory injury through decreasing nitroxidative stress and blocking TLR4 activation along with NF-KB phosphorylation in mice. J Ethnopharmacol 214: 292-300, 2018.

40. Wang C, Li Q and Li T: Dioscin alleviates lipopolysaccharide-induced acute lung injury through suppression of TLR4 signaling pathways. Exp Lung Res 46: 11-22, 2020.

41. Ouyang W, Zhou H, Liu C, Wang S, Han Y, Xia J and Xu F: 25-Hydroxycholesterol protects against acute lung injury via targeting MD-2. J Cell Mol Med 22: 5494-5503, 2018.

42. Shirey KA, Lai W, Scott AJ, Lipsky M, Mistry P, Pletneva LM, Karp CL, McAlees J, Gioannini TL, Weiss J, et al: The TLR4 antagonist Eritoran protects mice from lethal influenza infection. Nature 497: 498-502, 2013.

43. Cheng AM, Byrom MW, Shelton J and Ford LP: Antisense inhibition of human miRNAs and indications for an involvement of miRNA in cell growth and apoptosis. Nucleic Acids Res 33: 1290-1297, 2005.

44. Liu CJ,Fu X, Xia M,Zhang Q, Gu Z and Guo AY: MiRNASNP-v3: A comprehensive database for SNPs and disease-related variations in miRNAs and miRNA targets. Nucleic Acids Res 49 (D1): D1276-D1281, 2021.

45. Zhou X, Li X, Ye Y, Zhao K, Zhuang Y, Li Y, Wei Y and Wu M: MicroRNA-302b augments host defense to bacteria by regulating inflammatory responses via feedback to TLR/IRAK4 circuits. Nat Commun 5: 3619, 2014.

46. Wang B, Li X, Zhao G, Yan H, Dong P, Watari H, Sims M, Li W, Pfeffer LM, Guo Y and Yue J: MiR-203 inhibits ovarian tumor metastasis by targeting BIRC5 and attenuating the TGF $\beta$ pathway. J Exp Clin Cancer Res 37: 235, 2018.

47. Zhu M,Li Y and Sun K: MicroRNA-182-5p inhibits inflammation in LPS-treated RAW264.7 cells by mediating the TLR4/NF- $\kappa \mathrm{B}$ signaling pathway. Int J Clin Exp Pathol 11: 5725-5734, 2018

48. Goncalves Fernandes J, Morford LA, Harrison PL, Kompotiati T, Huang H, Aukhil I, Wallet SM and Macchion Shaddox L: Dysregulation of genes and microRNAs in localized aggressive periodontitis. J Clin Periodontol 47: 1317-1325, 2020.

49. Braga TV, Evangelista FCG, Gomes LC, Araujo SSDS, Carvalho MDG and Sabino AP: Evaluation of MiR-15a and MiR-16-1 as prognostic biomarkers in chronic lymphocytic leukemia. Biomed Pharmacother 92: 864-869, 2017.

50. Fennell D: MiR-16: Expanding the range of molecular targets in mesothelioma. Lancet Oncol 18: 1296-1297, 2017.

This work is licensed under a Creative Commons Attribution-NonCommercial-NoDerivatives 4.0 International (CC BY-NC-ND 4.0) License. 